\title{
Correlation between Gait Speed and Velocity of Center of Pressure Progression during Stance Phase in the Older Adults with Cognitive Decline: A Pilot Study
}

\author{
Hee-Chang Seon, PT, BS • Han-Suk Lee, PT, $\mathrm{PhD}^{1 \dagger} \cdot$ Man-Soo Ko, PT, $\mathrm{PhD}^{2} \cdot$ Sun-Wook Park, PT, PhD ${ }^{3}$ \\ Department of Physical Therapy, Graduate School, Eulji University, \\ ${ }^{1}$ Department of Physical Therapy, Eulji University, \\ ${ }^{2}$ Department of Physical Therapy, University of Texas Medical Branch, \\ ${ }^{3}$ Department of Physical Therapy, Samsung Medical Center
}

Received: October 20, 2020 / Revised: October 20, 2020 / Accepted: November 13, 2020

(C) 2020 J Korean Soc Phys Med

\section{| Abstract |}

PURPOSE: The progression of the center of pressure (COP) velocity of the stance phase may have important roles for predicting gait speed in older adults with cognitive decline. This study was conducted to identify the correlation between gait speed and the velocity of COP progression during the stance phase in older adults with cognitive decline.

METHODS: Forty adults aged 65 years or older (twenty participants without cognitive decline, 20 participants with cognitive decline) were recruited. The COP progression velocity was measured using an F-scan pressure-sensitive insole system. The stance phase was divided into four sub-stages. (loading response, mid-stance, terminal stance, and pre-swing). Gait speed, double support phase, and cadence were also measured. Correlations and multiple

$\dagger$ Corresponding Author : Han-Suk Lee

leehansuk21@hanmail.net, https://orcid.org/0000-0002-9336-0894 This is an Open Access article distributed under the terms of the Creative Commons Attribution Non-Commercial License (http://creativecommons.org/licenses/by-nc/3.0) which permits unrestricted non-commercial use, distribution, and reproduction in any medium, provided the original work is properly cited. regression analyses were performed.

RESULTS: Gait speed was associated with the COP progression velocity in midstance $(r=.719, \mathrm{p}<.05)$, cadence $(\mathrm{r}=.719, \mathrm{p}<.05)$ and the COP progression velocity in loading response velocity $(\mathrm{r}=.515, \mathrm{p}<.05)$ in older adults with cognitive decline. However, no correlation was found in older adults without cognitive decline. In multiple regression analysis using gait speed as a dependent variable, the COP progression velocity in midstance and cadence were significant predictors of gait speed, with the COP progression velocity being the most significant predictor.

CONCLUSION: The COP progression velocity is an important factor for predicting gait speed in older adults with cognitive decline, suggesting that the cognitive function influences gait speed and the velocity of COP progression.

Key Words: Gait, Pressure, Cognitive decline

\section{Introduction}

Gait speed reflects overall physical function [1] and decreases by $10-16 \%$ every 10 years in older adults [2]. Thus, a significant reduction of gait speed can be a risk factor for older adults predicting the physical health status 
and restriction of daily activities in the community [3-5]. Therefore, a common rehabilitation goal for frail older adults is to improve gait speed since gait speed is causally associated with muscle weakness, postural control, and cognitive deficits.

Biomechanically, gait speed is associated with the center of pressure (COP) movement on the foot during the stance phase of the gait cycle. The location of the COP represents the balance point where foot pressure changes from initial contact to pre-swing phase while in a steady-state gait [6]. Accordingly, the velocity of the COP movement corresponds to the forward progression of pressure changes on the plantar surface of the foot and the change of COP velocity can be a good indicator to describe the spatiotemporal characteristics of the stance phase in older adults with cognitive decline.

Fuchioka et al. [7] indicated that the gait speed could be affected by the COP velocity, so faster gait speed was associated with faster $\mathrm{COP}$ velocity during a steady-state gait. Specifically, this study highlighted that the COP velocity in the midstance of older adults was significantly associated with the gait speed. The mid-stance is a single limb support phase and requires a good body alignment by facilitating hip abductors and plantar flexors while walking. Thus, better posture and its subsequent joint torque improvement may effectively contribute to gait speed by enhancing the passive dynamics (e.g. inverted pendulum walking model) during a single limb support time. The COP velocity during the mid-stance phase can be a key component to explain slower gait speed in older adults with cognitive decline [8,9]. Lee et al [10] suggested that assessment of gait speed could be an effective clinical tool for predicting cognitive decline.

However, little attention has been given to clarify the association of gait speed and COP velocity in older adults with cognitive decline. Identifying the COP velocity in each stance phase can specify the treatment target for improvment of gait speed in older adults. Therefore, this study aimed at identifying the correlation between gait speed and COP velocity of gait cycles in healthy older adults as well as older adults with cognitive decline.

\section{Method}

\section{Subjects}

Forty adults aged 65 or older (20 in the normal group, 20 in the cognitive decline group) were recruited from the S Central Senior Welfare Center in Gyeonggi-do Seongnam-si from October 2018 to March 2019. The purpose of this study was explained in detail to all subjects, who voluntarily agreed to participate in the experiment. We calculated the sample size with G-power (effect size: .47 , power: $.95, a=.05$ ). All selected subjects were free of neurological disease, communication problems and walking problems, and each had the right foot as the dominant foot. The subjects included in the cognitive decline group were diagnosed by a doctor as high-risk with cognitive impairment. Participants in both groups were rescreened by a psychiatrist through the CERAD-K score for their suitability for inclusion in the normal group or the cognitive decline group. The investigation was approved by the Eulji University Institutional Review Board (EU18-72, EU19-19).

\section{Measurement}

The gait speed was measured using a 4-meter walking test (4 WMT, $\mathrm{r}=.85$ ) on a $6 \mathrm{~m}$ walking path. 4WMT was carried out thrice by the same tester. We observed that the third trial was the steady-state in both groups. Therefore, only the third trial was analyzed. The time required to walk the $4 \mathrm{~m}$ distance, except the first and last $1 \mathrm{~m}$ corresponding to the acceleration and deceleration period (seconds), was measured. Participants were instructed to take sufficient time to practice and to walk at a comfortable and normal pace. A Tekscan ${ }^{\circledR}$ F-Scan $®$ system (Tekscan Inc, South Boston, MA, USA, 2010) was 
(A)

(B)

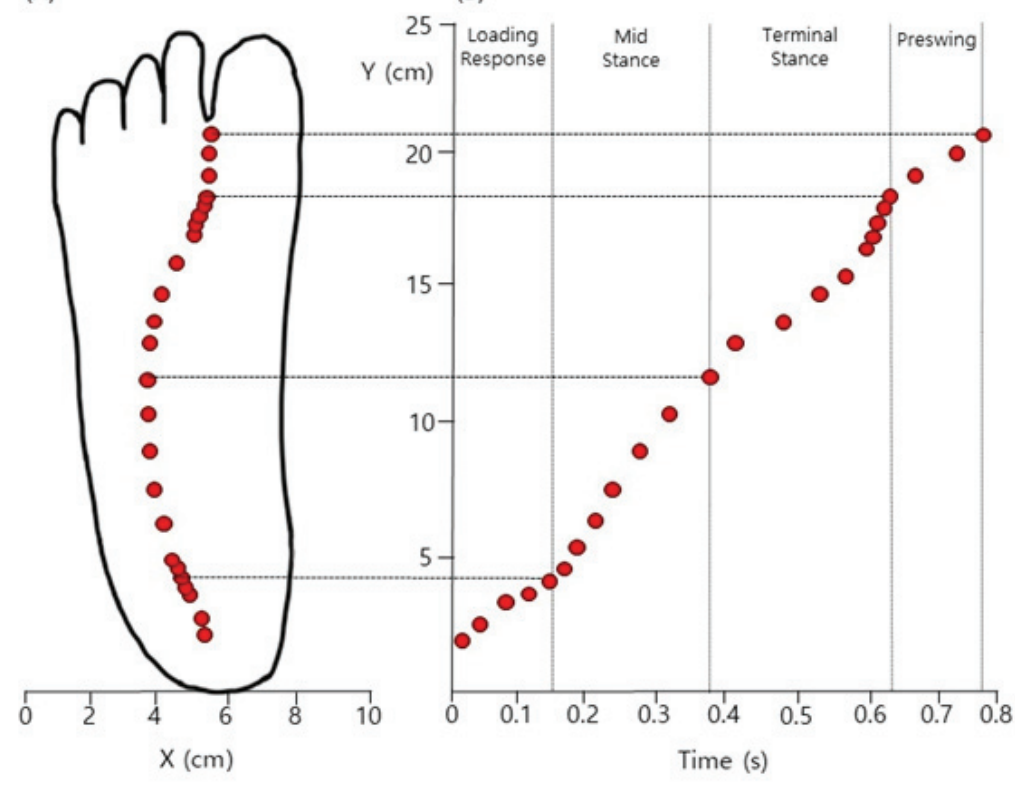

Fig. 1. The trajectory of the center of pressure (COP) in the foot. (A) The COP trajectory in $X-Y$ in the stance phase. (B) The COP movement is shown along the $\mathrm{Y}$-axis versus time.

used, and foot pressure was measured with a pressuresensing insole. Subjects wore shoes with insoles with amplifiers placed on the outside of both ankles. COP coordinates were calculated using the F-Scan software.

\section{Data analysis}

The first and last steps during the 4-WMT were excluded to analyze the steady-state of the gait. The COP progression velocity was calculated based on the COP coordinates according to the stance phase of the gait cycle period with the F-Scan software as shown in a previous study [6]. The COP coordinates ( $\mathrm{X}$ and $\mathrm{Y}$ ) were obtained by converting the COP measured by the transducer into American Standard Code for Information Interchange (ASCII) values using the F-Scan ${ }^{\circledR}$ software [11]. Figure. 1 shows a COP trajectory of the foot. The stance phase was divided into loading response, mid-stance, terminal stance, and preswing to calculate the COP progression velocity.

\section{Statistical analysis}

The general characteristics of the participants were analyzed using descriptive statistics. The assumption of normality was tested using Shapiro-Wilk tests and it was ascertained that variables followed a normal distribution. An independent t-test was conducted to compare the general characteristics of the subjects. Pearson's correlation coefficient was used to investigate the correlation between gait speed and walking variables including the COP velocity during the four-sub-stance phases, double supporting time, and cadence. The multiple regression analysis was used to predict the factors that affect gait speed. The gait speed was used as the dependent variable. Age, double support stance, cadence, COP velocity of the gait phase were taken as independent variables. Statistical processing was performed using SPSS for Windows (version 18) and p-values $<.05$ were considered significant. 


\section{Results}

The mean age of the subjects was $76.80 \pm 5.37$ years in the normal group and $77.50 \pm 6.81$ years in the cognitive decline group. There were significant differences between groups in the cognitive function score (measured by KMMSE) $(p<.05)$. The score was $26.05 \pm 2.33$ in the normal group and $20.65 \pm 3.23$ in the cognitive decline group. Among the gait variables, the gait speed, was significantly different between the two groups, $.78 \pm .15$ $\mathrm{m} / \mathrm{s}$ in the normal group and $.58 \pm .18 \mathrm{~m} / \mathrm{s}$ in the cognitive decline group $(\mathrm{p}<.05)$. The time taken in the double support phase, was also significantly different between the two groups ( $\mathrm{p}<.05), .12 \pm .02 \mathrm{sec}$ in the normal group and $.18 \pm .05 \mathrm{sec}$ in the cognitive decline group). Also, the $\mathrm{COP}$ velocity of midstance from the COP velocity of gait phase, was significantly different between groups, $(.30 \pm$ $.09 \mathrm{~m} / \mathrm{s}$ in the normal group and $.21 \pm .10 \mathrm{~m} / \mathrm{s}$ in the cognitive decline group $(\mathrm{p}<.05)$ (Table 1).

There was a strong positive correlation between the midstance COP velocity $(\mathrm{r}=.719, \mathrm{p}<.05)$, cadence $(\mathrm{r}$ $=.719, \mathrm{p}<.05)$ and the gait speed in the cognitive decline group. There was a moderate positive correlation between the loading response COP velocity $(\mathrm{r}=.515, \mathrm{p}<.05)$ and gait speed, but there was a moderate negative correlation between the double support phase $(\mathrm{r}=-.568$, $\mathrm{p}<.05$ ) and the gait speed (Table 2). However, there was no such correlation in the normal group with gait speed (Table 2). Specifically, COP velocity of the midstance was a variable $(p=.059)$ that influenced the gait speed in the cognitive decline group (Table 3).

The multiple regression analysis of the cognitive decline

Table 1. Characteristics of the Participants

\begin{tabular}{|c|c|c|c|}
\hline Characteristics & $\begin{array}{l}\text { Old Adults without Cognitive Decline } \\
\qquad(\mathrm{n}=20)\end{array}$ & $\begin{array}{l}\text { Old Adults with Cognitive Decline } \\
\qquad(\mathrm{n}=20)\end{array}$ & $\mathrm{p}$ \\
\hline \multicolumn{4}{|c|}{ Physical Characteristics } \\
\hline \multirow{2}{*}{$\begin{array}{c}\text { Male (n) } \\
\text { Female (n) }\end{array}$} & 4 & 6 & \\
\hline & 16 & 14 & \\
\hline Age (y) & $76.80 \pm 5.37$ & $77.50 \pm 6.81$ & .720 \\
\hline Height $(\mathrm{cm})$ & $153.71 \pm 8.16$ & $155.73 \pm 7.58$ & .422 \\
\hline Weight (kg) & $55.62 \pm 7.67$ & $56.56 \pm 10.48$ & .747 \\
\hline Body Mass Index $\left(\mathrm{kg} / \mathrm{m}^{2}\right)$ & $23.49 \pm 2.12$ & $23.18 \pm 2.87$ & .706 \\
\hline K-MMSE (score) & $26.05 \pm 2.33$ & $20.65 \pm 3.23$ & $.000^{*}$ \\
\hline \multicolumn{4}{|c|}{ Gait Variables } \\
\hline Gait Speed $(\mathrm{m} / \mathrm{s})$ & $.78 \pm .15$ & $.58 \pm .18$ & $.000^{*}$ \\
\hline Double Support Phase (s) & $.12 \pm .02$ & $.18 \pm .05$ & $.000^{*}$ \\
\hline Cadence (steps/min) & $98.53 \pm 20.85$ & $93.72 \pm 18.09$ & .441 \\
\hline \multicolumn{4}{|c|}{ The Velocity of COP Progression in the Stance Phase } \\
\hline Loading Response (m/s) & $.19 \pm .08$ & $.15 \pm .08$ & .184 \\
\hline Mid-Stance $(\mathrm{m} / \mathrm{s})$ & $.30 \pm .09$ & $.21 \pm .10$ & $.005^{*}$ \\
\hline Terminal Stance $(\mathrm{m} / \mathrm{s})$ & $.37 \pm .12$ & $.36 \pm .25$ & .864 \\
\hline Pre-Swing (m/s) & $.22 \pm .07$ & $.20 \pm .01$ & .160 \\
\hline
\end{tabular}

$\mathrm{COP}=$ Center of pressure, $\mathrm{s}=$ Second, ${ }^{*} \mathrm{p}<.05$ 
Table 2. Correlations between Gait Speed and the Velocity of COP Progression duringthe Stance Phase in Older Adults with and without Cognitive Impairment

\begin{tabular}{|c|c|c|c|c|c|c|}
\hline Variable & $\begin{array}{c}\text { Double } \\
\text { Support } \\
\text { Phase }\end{array}$ & Cadence & $\begin{array}{l}\text { Loading } \\
\text { Response } \\
\text { Velocity }\end{array}$ & $\begin{array}{l}\text { Mid-Stance } \\
\text { Velocity }\end{array}$ & $\begin{array}{l}\text { Terminal } \\
\text { Stance } \\
\text { Velocity }\end{array}$ & $\begin{array}{l}\text { Pre-Swing } \\
\text { Velocity }\end{array}$ \\
\hline $\begin{array}{l}\text { Gait speed for older adults with cognitive } \\
\text { impairment }\end{array}$ & $-.568^{* *}$ & $.719^{* *}$ & $.515^{*}$ & $.748^{* *}$ & -.179 & -.149 \\
\hline $\begin{array}{l}\text { Gait speed older adults without cognitive } \\
\text { impairment }\end{array}$ & -.392 & -.114 & .023 & .429 & .081 & .133 \\
\hline
\end{tabular}

Table 3. Multiple Regression Analysis with Gait Speed as a Dependent Variable in Older Adults with Cognitive Impairment

\begin{tabular}{ccccc}
\hline Independent Variable & $\beta$ & SE & Adjusted $\beta$ & $\mathrm{p}$ \\
\hline Mid Stance COP Velocity & .846 & .330 & .486 & $<.020^{*}$ \\
Cadence & .004 & .002 & .401 & $<.049^{*}$ \\
\hline
\end{tabular}

$\mathrm{R}=.807$, Adjusted $\mathrm{R}^{2}=.610,{ }^{*} \mathrm{p}<.05$

group showed $61 \%$ explanatory power indicating that the midstance COP velocity (adjusted B: .486) and the cadence (adjusted B: .401) can affect the gait speed. Additionally, the midstance COP velocity can affect the gait speed to a greater extent compared to the cadence (Table 3).

\section{Discussion}

In this study, we verified the correlation between gait speed and COP velocity of gait cycles in healthy older adults and those with cognitive decline. We found that there was no correlation between gait speed and COP velocity, age, cadence, double support phase (DSP) in the elderly without cognitive decline. In contrast, in the elderly with cognitive decline, there was a correlation between the midstance COP velocity, cadence, and gait speed. The most important finding was that COP velocity of midstance can affect gait speed.

This study represents the COP progression characteristics in older adults with a cognitive decline through comparison with older adults without cognitive decline. Older adults with cognitive decline had a lower cadence, gait speed and duration of double support which is consistent with previous studies. Moreover, older adults with cognitive decline had a slower COP progression velocity in all four sub-stance gait phases compared to older adults without cognitive decline.

The slower COP velocity means that longer stance phase and diminishing forward body progression occurred in older adults with cognitive decline. Thus, older adults with cognitive decline had limited forward body progression. The forward body progression can be controlled by the plantar flexors of the ankle such as the gastrocnemius and the soleus. The COP path characteristics could represent the foot function dynamics [12]. De Cock et al. [13] suggested that COP displacement could be a potential measure of foot function. Therefore, the slower COP velocity is related to the plantar flexors that control the foot function and forward body progression.

The COP velocity in midstance did not have a significant correlation with the gait speed in the elderly without cognitive decline $(p=.059)$. The reason for this result 
could be the small sample size. Therefore, it is necessary to analyze this parameter in a large sample size in future studies. In contrast, we found that the COP velocity of midstance and gait speed in older adults with cognitive decline were significantly correlated $(p<.05)$. The slower gait speed led to lower COP displacement velocity during midstance in the elderly without cognitive decline. Therefore, cognitive function may affect the COP velocity in midstance.

It is probable that the gait speed is affected by a deficit of the prefrontal lobe [14]. Gait is a combination of the interaction of executive functions, cognition, emotion, and sensation [15]. The prefrontal lobe, which plays a major role in executive functions, is functionally connected to the hippocampus that affects cognitive decline [16]. There is a positive correlation between the K-MMSE score and both the frontal lobe and the hippocampus. Therefore, a decrease in the MMSE score suggests a decrease in the function of the frontal lobe and hippocampal regions $[17,18]$, thereby also suggesting that older adults with cognitive decline who may have a damage in the prefrontal lobe and hippocampus may show gait disorders [19], specially COP velocity in midstance.

Recently, Jang et al reported that there was a moderate positive correlation $(\mathrm{r}=.491)$ between cognitive function (K-MMSE) and hip abduction muscle strength [20]. This study indicated that the weakness of hip abduction was remarkable in older adults with cognitive decline. The hip abductor is an important factor in restoring balance and independent gait [21]. In general, the hip abductor is the most important muscle for stabilizing the pelvis during the mid-stance phase while walking [22-24]. A weakness of the hip abductor could cause a pelvic drop on the contralateral side during the mid-stance phase [25], and this might restrict the contralateral mid-swing phase as well. Thus, the mid-stance could be prolonged with restricted forward progression while walking. The COP velocity could be slower under such constrained passive dynamics during the mid-stance.

During mid-stance, the center of mass moves into the base of support to gain stability. If the person has a weakness in the gluteus medius, he or she may show excessive lateral trunk bending to compensate for the pelvic drop on the contralateral side. This compensation can drive the movement of COP into the base of support. Our study observed the pelvic drop on the contralateral side using video analysis, but this gait deviation was not linked with the excessive lateral trunk bending in older adults with cognitive decline.

Moreover, this study found a positive correlation between the COP velocity during loading response and the gait speed in older adults in the cognitive decline group. Interestingly, we found that the COP velocity during loading response in older adults with cognitive decline was slower than in older adults without cognitive decline.

Generally, foot and ankle characteristics contribute to the loading response. Older adults show a decreasing range of motion in ankle plantar-dorsiflexion that governs the magnitude of force and pressure under the heel. The Min chi Chiu study [12] reported that the elderly had less contact period with fast COP velocity during initial contact due to significant heel eversion (more medial COP shift). In contrast in the elderly without cognitive decline, this study revealed the contact period was longer. This could be due to the diminishing ankle plantar-dorsiflexion movement [26].

During the loading response phase, proprioceptive stimulation comes in, and proper processing is required to maintain walking stability [27]. However, this kind of processing can be slower due to cognitive impairment [28]. Therefore, the elderly without cognitive decline show slower COP progression velocity. These findings imply that the elderly with cognitive decline adopt different strategies to eliminate impact thus creating stability in the loading response phase.

This study has several limitations. The sample size is small, so it is difficult to generalize the results to the entire 
elderly population. In addition, we recruited only adults with mild cognitive impairment, not those with severe cognitive decline. Lastly, since kinematic analysis through video was not used, there is a limitation on the interpretation of results as well.

\section{Conclusion}

The main purpose of this study was to identify the association between gait speed and COP progression velocity of stance phase in older adults with and without cognitive decline. Among the stance phases, the velocity of COP progression in mid-stance was observed to be related to gait speed in older adults with cognitive decline. We conclude that cognitive functions affect the velocity of COP progression and gait speed. It is therefore necessary to take this into account during clinical evaluation of older adults with various cognitive impairments in the future.

\section{Acknowledgments}

This work was supported by the National Research Foundation of Korea (NRF) grant funded by the Korea government (MSIT) (No. NRF-2018R1D1A1B07047562).

\section{References}

[1] Guralnik JM, Ferrucci L, Simonsick EM, et al. Lower-Extremity Function in Persons over the Age of 70 Years as a Predictor of Subsequent Disability. N Engl J Med. 1995;332(9):556-62.

[2] Brach JS, FitzGerald S, Newman AB, et al. Physical Activity and Functional Status in Community-Dwelling Older Women: A 14-Year Prospective Study. Archives of Internal Medicine. 2003;163(21):2565-71.

[3] Cesari M, Kritchevsky SB, Penninx BWHJ, et al. Prognostic Value of Usual Gait Speed in Well-Functioning Older People-Results from the Health, Aging and Body
Composition Study. J Am Geriatr Soc. 2005;53(10): 1675-80.

[4] Montero-Odasso M, Schapira M, Soriano ER, et al. Gait Velocity as a Single Predictor of Adverse Events in Healthy Seniors Aged 75 Years and Older. The Journals of Gerontology: Series A. 2005;60(10):1304-9.

[5] Studenski S, Perera S, Patel K, et al. Gait Speed and Survival in Older Adults. JAMA. 2011;305(1):50-8.

[6] Schmid M, Beltrami G, Zambarbieri D, et al. Centre of pressure displacements in trans-femoral amputees during gait. Gait Posture. 2005;21(3):255-62.

[7] Fuchioka S, Iwata A, Higuchi Y, et al. The Forward Velocity of the Center of Pressure in the Midfoot is a Major Predictor of Gait Speed in Older Adults. International Journal of Gerontology. 2015;9(2):119-22.

[8] Buracchio T, Dodge HH, Howieson D, et al. The trajectory of gait speed preceding mild cognitive impairment. Archives of neurology. 2010;67(8):980-6.

[9] Best JR, Liu-Ambrose T, Boudreau RM, et al. An evaluation of the longitudinal, bidirectional associations between gait speed and cognition in older women and men. Journals of Gerontology Series A. 2016;71(12): 1616-23.

[10] Lee HS, Ko M, Park SW, et al. Concurrent validity of the Groningen Meander Walking and Timed Up and Go tests in older adults with dementia. Physiotherapy theory and practice. 2019:1-6.

[11] Baek NJ, Lim MS. The Path of Center of Pressure (COP) of the Foot during Walking. Annals of Rehabilitation Medicine. Journal of the Korean Academy of Rehabilitation Medicine. 1997;21(4):762-71.

[12] Chiu MC, Wu HC, Chang LY, et al. Center of pressure progression characteristics under the plantar region for elderly adults. Gait Posture. 2013;37(3):408-12.

[13] De Cock A, Vanrenterghem J, Willems T, et al. The trajectory of the centre of pressure during barefoot running as a potential measure for foot function. Gait Posture. 2008;27(4):669-75. 
[14] Yogev-Seligmann G, Hausdorff JM, Giladi NJ. The role of executive function and attention in gait. Movement disorders: official journal of the Movement Disorder Society. 2008;23(3):329-42.

[15] Snijders AH, Van De Warrenburg BP, Giladi N, et al. Neurological gait disorders in elderly people: clinical approach and classification. The Lancet Neurology. 2007;6(1):63-74.

[16] Pugh KG, Lipsitz LA. The microvascular frontalsubcortical syndrome of aging. Neurobiology of aging. 2002;23(3):421-31.

[17] Golomb J, De Leon M, George A, et al. Hippocampal atrophy correlates with severe cognitive impairment in elderly patients with suspected normal pressure hydrocephalus. Journal of Neurology, Neurosurgery \& Psychiatry. 1994;57(5):590-3.

[18] Choi J, Ku B, You YG, et al. Resting-state prefrontal EEG biomarkers in correlation with MMSE scores in elderly individuals. Scientific reports. 2019;9(1):1-15.

[19] Malouin F, Richards CL, Jackson PL, et al. Brain activations during motor imagery of locomotor related tasks: A PET study. Human brain mapping. 2003;19(1): 47-62.

[20] Jang IY. Association Between Muscle Strength and Cognitive Function in Elderly Koreans. Doctor's Degree. CHA University. 2020.

[21] Kirker S, Simpson D, Jenner J, et al. Stepping before standing: hip muscle function in stepping and standing balance after stroke. Neurosurgery \& Psychiatry. 2000; 68(4):458-64.

[22] Friel K, McLean N, Myers C, et al. Ipsilateral hip abductor weakness after inversion ankle sprain. Journal of athletic training. 2006;41(1):74.

[23] Jacobs CA, Uhl TL, Mattacola CG, et al. Hip abductor function and lower extremity landing kinematics: sex differences. Journal of athletic training. 2007;42(1):76.

[24] Widler KS, Glatthorn JF, Bizzini M, et al. Assessment of hip abductor muscle strength. A validity and reliability study. JBJS. 2009;91(11):2666-72.

[25] Hollman JH, Kolbeck KE, Hitchoock JL, et al. Correlations between hip strength and static foot and knee posture. Journal of Sport Rehabilitation. 2006;15(1):12-23.

[26] Boyer KA, Andriacchi TP, Beaupre GS, et al. The role of physical activity in changes in walking mechanics with age. Gait Posture. 2012;36(1):149-53.

[27] Kim IB, Park TS, Kang JH. Comparison of Barefoot and Shod Gait Cycle for Adult Women. JJoCflT. 2018;8(1):9-14.

[28] Viscogliosi G, Di Bernardo M, Ettorre E, et al. Handgrip strength predicts longitudinal changes in clock drawing test performance. An observational study in a sample of older non-demented adults. The journal of nutrition, health \& aging. 2017;21(5):593-6. 